\title{
Preventive Effects of Antioxidants on Muscle Atrophy Induced by Ischemic Reperfusion
}

\author{
NAMIKO UMEI ${ }^{1,2)}$, TAKEYA ONO ${ }^{1)}$, SADAAKI OKI ${ }^{1)}$, AKIRA OTSUKA ${ }^{1)}$, \\ HirOshi OTAO ${ }^{1)}$, NORIO MUTO ${ }^{2)}$ \\ 1) Department of Physical Therapy, Faculty of Health and Welfare, Prefectural University of Hiroshima: \\ 1-1 Gakuen Machi, Mihara City, Hiroshima, 723-0053 Japan. \\ TEL: +81848-60-1225, E-mail: umei@pu-hiroshima.ac.jp \\ 2) Program in Biological System Sciences, Graduate School of Comprehensive Scientific Research, \\ Prefectural University of Hiroshima
}

\begin{abstract}
Purpose] This study aimed to determine whether muscle atrophy induced by ischemic reperfusion injury in rats can be prevented by the administration of antioxidants. [Subjects] Rats were randomly divided into three groups: the non-treated group, vitamin $\mathrm{C}$ group, and vitamin $\mathrm{E}$ group. [Methods] Ischemia was induced in the right lower limb a pressure of $300 \mathrm{mmHg}$ for 90 minutes.Vitamin injections were administered six times from just prior to the induction of ischemia until 60 hours after the reperfusion. The relative weight ratio of the soleus muscle and the length of the soleus muscle fiber cross-section minor axes were used for the evaluation of muscle atrophy, and biochemical analyses of the creatine phosphokinase and total glutathione levels were also performed. [Results] The relative weight ratios of the soleus muscle and the lengths of the soleus muscle fiber cross-section minor axis in the vitamin $\mathrm{C}$ group and the vitamin E group were significantly greater than those in the non-treated group. In comparison with the non-treated group, the total glutathione level showed a tendency to be higher in the vitamin $\mathrm{C}$ group and was significantly higher in the vitamin E group. [Conclusion] In this study, using an experimental rat model, we confirmed that antioxidants prevent the muscle atrophy induced by ischemic reperfusion.
\end{abstract}

Key words: Ischemic reperfusion, Muscle atrophy, Antioxidant

(This article was submitted Jan. 31, 2011, and was accepted Feb. 25, 2011)

\section{INTRODUCTION}

The use of tourniquets on the limbs in cosmetic surgery can produce ischemia-related changes. Ischemic reperfusion is known to induce several disorders such as inflammation around blood vessels after blood flow is reinstated ${ }^{1)}$. Knight examined the effects of 24 hours reperfusion after two-hours tourniquet-induced ischemia and found that muscle wet weight was significantly increased after one-hour reperfusion and peaked 16 hours later. The increase in muscle wet weight remained significant at 24 hours after the reperfusion. In addition, as the cell death that occurred after the ischemic reperfusion was accompanied by necrosis, total glutathione levels decreased and the number of neutrophils in the muscle increased after reperfusion ${ }^{2)}$. The cell necrosis observed during the ischemic reperfusion was induced by reactive oxygen species (ROS) generated by the supply of oxygen after reperfusion. Increased levels of ROS are a critical causal factor of tissue disorders ${ }^{2,3)}$. In our previous study, we examined the tissue changes that occur after ischemic reperfusion using an experimental animal model and confirmed that muscle atrophy and edema occur simultaneously at 72 hours after ischemic reperfusion.
This study aimed to determine whether the muscle atrophy induced by ischemic reperfusion injury in rats can be prevented by antioxidant treatment.

\section{SUBJECTS AND METHODS}

\section{Subjects}

Fifteen female Wistar rats (eight weeks old) were used. The rats were randomly divided into three groups of five rats each: the non-treated group, vitamin $\mathrm{C}$ group, and vitamin $\mathrm{E}$ group. The experiments were conducted in accordance with our university's Guidelines for Animal Experimentation and the U.S. National Institute of Health Guidelines.

\section{Methods}

The rats were allowed free access to a standard diet and water in their cages. Ischemia was induced with a DC1.6 tourniquet finger cuff, and a Rapid Cuff Inflator with an AG101 air source (D.E. Hokanson, USA) was used to apply pressure. The rats were anaesthetized with sodium pentobarbital (40 $\mathrm{mg} / \mathrm{kg}$ b.w.), and ischemia was induced in the right lower limb at a pressure of $300 \mathrm{mmHg}$ for 90 minutes. 
The vitamin $\mathrm{C}$ group was intraperitoneally injected with $40 \mathrm{mg} / \mathrm{kg}$ b.w. ascorbic acid (Fuso, Osaka, Japan). The vitamin E group was intramuscularly injected with $30 \mathrm{mg} /$ $\mathrm{kg}$ b.w. tocopherol acetate (Eisai, Tokyo, Japan). All injections were performed six times (once every 12 hours) from just prior to the ischemia procedure until 60 hours of reperfusion had elapsed. The dosages were derived from Kondo's ${ }^{4)}$ manual and research.

All rats were weighed at the beginning and the end of the experiment. The rats were anaesthetized with sodium pentobarbital ( $40 \mathrm{mg} / \mathrm{kg} \mathrm{b.w.)} \mathrm{after} \mathrm{being} \mathrm{weighed} \mathrm{at} \mathrm{the} \mathrm{end}$ of the experiment. Then, the rats were exsanguinated by cutting the abdominal aorta, and the soleus muscle of the right hind leg was extracted by cutting it between the top of the calcaneus and the bottom of the fibula head. The wet weight of the soleus muscle was measured with a precise balance immediately after the mice had been sacrificed. The relative weight ratio of the soleus muscle was calculated as the ratio of the wet weight of the soleus muscle to the body weight of the rat.

After its wet weight had been measured, the soleus muscle was divided in half and embedded in tragacanth. The excised soleus muscle was then rapidly frozen by immersing it in a liquid nitrogen bath. Sections, $10 \mathrm{~m}$ thick, were prepared using a cryostat microtome and stained with hematoxylin and eosin (H\&E staining). All sections were photographed using a digital camera and the muscle fiber cross-section minor axis was measured microscopically. The images were transferred to a computer, and the fiber cross-section minor axis of the muscle was measured using image analysis software (Image ProPlus 6.2J,USA). The fiber cross-section minor axes of more than 200 muscle fibers were measured, and the mean value was calculated.

Creatine phosphokinase (CPK) was assayed by the JSCC standardization method. Abdominal aortic blood was drawn from each rat at the time of sacrifice. After being left at room temperature for more than 15 minutes, the blood was centrifuged at $4^{\circ} \mathrm{C}$ for 15 minutes at $3,500 \mathrm{rpm}$. After separating the supernatant fluid (serum), it was used for the measurement of CPK activity.

Total glutathione (GSH) was assayed using a total glutathione fixed-quantity kit (Dojindo, Kumamoto, Japan). The muscle tissue was washed with an isotonic sodium chloride solution. Homogenization was performed with 5\% sulfosalicylic acid, followed by centrifugation at $4{ }^{\circ} \mathrm{C}$ for 20 minutes at 3,500 rpm. After separating the supernatant fluid, it was used to measure the GSH level.

Group data were analyzed using a multiple comparison test (SPSS15.0J for Windows, SPSS Inc.), and p values $<0.05$ were considered to be significant.

\section{RESULTS}

As one soleus muscle sample in the non-treated group was incomplete, the final data for the relative soleus muscle weight ratios and the soleus muscle fiber cross-section minor axis length in the non-treated group were obtaining from 4 specimens.

The mean soleus muscle relative weight ratios were 0.43
Table 1. Effects of antioxidants on skeletal muscle tissue $($ mean \pm SD)

\begin{tabular}{lcc}
\hline Group & $\begin{array}{c}\text { Relative weight ratio } \\
(\mathrm{mg} / \mathrm{g} \text { body weight })\end{array}$ & $\begin{array}{c}\text { Fiber cross-section } \\
\text { minor axis }(\mu \mathrm{m})\end{array}$ \\
\hline Non- treated group & $0.43 \pm 0.12$ & $37.7 \pm 1.7$ \\
Vitamin C group & $0.51 \pm 0.29^{\mathrm{a}}$ & $44.5 \pm 2.9^{\mathrm{b}}$ \\
Vitamin E group & $0.51 \pm 0.32^{\mathrm{a}}$ & $44.2 \pm 3.1^{\mathrm{b}}$ \\
\hline
\end{tabular}

${ }^{a}$ : non treated group; $p<0.01{ }^{b}$ : non treated group; $p<0.05$.

Table 2. Effects of antioxidants on serum $\mathrm{CPK}$ and tissue total GSH levels (mean \pm SD)

\begin{tabular}{lcc}
\hline Group & CPK $(\mathrm{IU} / \mathrm{L})$ & $\mathrm{GSH}(\mu \mathrm{mol} / \mathrm{L})$ \\
\hline Non- treated group & $889.4 \pm 365.5$ & $195.2 \pm 51.1$ \\
Vitamin C group & $587.6 \pm 368.9$ & $259.1 \pm 71.2$ \\
Vitamin E group & $383.2 \pm 81.0$ & $287.8 \pm 84.7^{\text {a }}$ \\
\hline
\end{tabular}

${ }^{a}$ : non treated group; $\mathrm{p}<0.05$.

$\mathrm{mg} / \mathrm{g} \mathrm{b.w}$. for the non-treated group, $0.51 \mathrm{mg} / \mathrm{g} \mathrm{b.w}$. for the vitamin C group, and $0.52 \mathrm{mg} / \mathrm{g}$ b.w. for the vitamin $\mathrm{E}$ group. The soleus muscle relative weight ratios in the vitamin $\mathrm{C}$ group and the vitamin E group were significantly larger than those in the non-treated group (Table 1).

The mean length of the soleus muscle fiber cross-section minor axis of the non-treated group was $37.73 \mu \mathrm{m}$; that of the vitamin C group was $44.49 \mu \mathrm{m}$; and that of the vitamin E group was $44.18 \mu \mathrm{m}$. The soleus muscle fiber crosssection minor axes in the vitamin $\mathrm{C}$ group and vitamin $\mathrm{E}$ group were significantly longer than those in the non-treated group (Table 1).

The mean serum CPK level in the non-treated group was 776.50 IU/L; that in the vitamin C group was $587.60 \mathrm{IU} / \mathrm{L}$; and that in the vitamin E group was $383.20 \mathrm{IU} / \mathrm{L}$. Compared with the non-treated group, the CPK level showed a tendency to be lower in the vitamin $\mathrm{C}$ group and the vitamin E group (Table 2).

The mean total tissue GSH level of the non-treated group was $195.24 \mu \mathrm{mol} / \mathrm{L}$; that of the vitamin $\mathrm{C}$ group was 259.08 $\mu \mathrm{mol} / \mathrm{L}$; and that of the vitamin E group was $287.81 \mu \mathrm{mol} /$ L. Compared with the non-treated group, the total GSH level tended to be higher in the vitamin $\mathrm{C}$ group and was significantly higher in the vitamin E group (Table 2).

\section{DISCUSSION}

The atrophying of skeletal muscle is caused by a decrease in the diameter of the muscle fibers and/or a decrease in the number of muscle fibers. It has been proposed that the proteins present in muscle cells constitute a lysosome system, a calpain system, and a ubiquitin-proteasome system. Among them, the ubiquitin-proteasome system is activated by physiological stress, such as ischemia ${ }^{3)}$. Reoxygenation after reperfusion generates a large quantity of ROS in the ischemic region, and the ROS generated by white blood corpuscle permeation can also have damaging effects $^{1,5)}$. ROS increase oxidative stress and promote 
skeletal muscle proteolysis. Nakashima reported that oxidative stress causes protein oxidation, and as a result, proteasomes, proteolytic enzyme complexes, were activated, which promote proteolysis. This was confirmed by examining the mechanism of the skeletal muscle proteolysis induced by oxidative stress in a culture experiment using skeletal muscle cells derived from chick embryos $^{6)}$. Thus, oxidative stress is responsible for the skeletal muscle atrophy that occurs after ischemia/ reperfusion, and it is concluded that it is important to alleviate oxidative stress to prevent amyotrophy. Kondo reported that the injection of vitamin E resulted in a decrease in immobility-induced muscle atrophy, while oxidative stress increased during the muscle atrophy recovery period $^{4)}$.

In this study, vitamin $\mathrm{C}$ and vitamin $\mathrm{E}$ were used as antioxidants to remove oxidative molecules. Vitamin $\mathrm{C}$ (ascorbic acid) and vitamin $\mathrm{E}$ are chain reaction-abrogating type antioxidants and are able to scavenge ROS to prevent oxidative stress. Vitamin $\mathrm{C}$ is distributed in both the extracellular fluid and the cytoplasm of muscle cells. Its two hydroxyl groups at the C-2 and C-3 positions participate in stabilization of radicals before they can attack the cellular membrane. In contrast, fat-soluble vitamin E exerts antioxidative effects in biomembranes and it binds to peroxyl radicals, which facilitate oxidative chain reactions ${ }^{7-9}$ ).

In the present study, the relative soleus muscle weight ratio was significantly higher and the soleus muscle fiber cross-section minor axis was significantly longer in the vitamin $\mathrm{C}$ group and vitamin E group than in with the nontreated group. This suggests that the muscle atrophy caused by ischemia/reperfusion can be prevented with antioxidant treatment. In comparison with the non-treated group, the total GSH level was significantly higher in the vitamin E group and showed a tendency to be higher in the vitamin $\mathrm{C}$ group, indicating that antioxidants decrease oxidative stress. GSH is one of the antioxidants found in the human body. Total GSH decreases when oxidative stress increases. It is clear that the total GSH of muscle cells plays a supporting role in protecting these cells from oxidative stress. The reason why the total GSH level of the non-treated group was low in our study is that GSH was used for the removal of ROS. In comparison with the non-treated group, the CPK level tended to be lower in the vitamin $\mathrm{C}$ and vitamin $\mathrm{E}$ groups. CPK suppresses the permeation of white blood corpuscles. Yamamura reported that edaravone, a medicinal drug with a similar action to CPK, improved the number of surviving muscle cells in an experiment involving 5-hr ischemia followed by 5 -hr reperfusion. In addition, the CPK level in the treated group was lower than that in the nontreated group, although the difference was not significant ${ }^{10}$. On the other hand, Irie reported an experiment involving 4$\mathrm{hr}$ ischemia followed by $24-\mathrm{hr}$ reperfusion during aboveknee amputation without blood vessel removal and found that the treated group showed significantly lower CPK levels ${ }^{11)}$. The present study's results were similar to those of Irie's group.

In this study, it was confirmed using an experimental rat model that antioxidant treatment can prevent the muscle atrophy induced by ischemic reperfusion. Wistar rats produce large quantities of endogenous ascorbic acid, and its normal level is much higher than that of endogenous vitamin E. Therefore, the injection of vitamin $\mathrm{E}$ is an efficient method of inducing antioxidative effects. In addition, the antioxidative ability of vitamin $\mathrm{E}$ may be reinforced by the coexistence of ascorbic acid. Consequently, vitamin $\mathrm{E}$ was a more potent antioxidant than vitamin $\mathrm{C}$ in the present study.

Further studies should be carried out to establish the appropriate doses of vitamins $\mathrm{C}$ and $\mathrm{E}$, including combination treatments of both vitamins.

\section{REFERENCES}

1) Hida Y, Kondo S: Ischemia-reperfusion Injury. Surgery Frontier, 2007, 14: 67-73 (in Japanese).

2) Knight KR, Messina A, Hurley JV, et al.: Muscle cells become necrotic rather than apoptotic during reperfusion of ischaemic skeletal muscle. Int J Exp Pathol, 1999, 80: 169-175.

3) Yoshioka T, Goto K, Kawasaki K: Molecular mechanism for muscle atrophyintracellular Proteolysis and it's regulation. Journal of Clinical and Experimental Medicine, 2000, 193 : 598-603 (in Japanese).

4) Kondo H, Kodama J, Kishibe T, et al.: Oxidative stress during recovery from muscle atrophy. European Biochemical Societies, 1993, 326: 189-191.

5) Inoue M: From active oxygen and a condition of a patient -disease model to the bedside. Tokyo: Japan Scientific Societies Press, 1992, p39 (in Japanese).

6) Nakashima K, Ishida A, Yamazaki M, et al.: Leucine suppresses myofibrillar proteolysis by down-regulating ubiquitin-proteasome pathway in chick skeletal muscles. Biochemical and Biophysical Research Communications, 2005, 336: 660-666.

7) Yoshikawa $\mathrm{T}$, Takahashi S, Kondo M: Ischemia-reperfusion injury and vitamin E. Vitamins, 1992, 66: 72-89 (in Japanese).

8) Sato N, Sato K: Vitamin C/E. Inflammation and Immunity, 2008, 16: 540544 (in Japanese).

9) Fukuzawa K: Foundations of vitamin E. Modern Physician, 2007, 27: 1262 1264 (in Japanese).

10) Yamamura M, Miyamoto $Y$, Mitsuo M, et al.: Suppression of rat lower extremity postoperative reperfuusion injury with edaravone. Journal of Angiology, 2006, 15: 34-36.

11) Irie $H$, Kato $T$, Ikebe $K$, et al.: Antioxidant effect of mci-186, a new freeradical scavenger, on ischemia-reperfusion injury in a rat hindlimb amputation model. Journal of Surgical Research, 2004, 120: 312-319. 